\title{
Institutional delivery service utilization in Woldia, Ethiopia
}

\author{
Worku Awoke $e^{1}$, Jemal Muhammed ${ }^{2}$, Gedefaw Abeje ${ }^{1}$ \\ ${ }^{1}$ Bahir Dar University, College of medicine and Health Sciences, Ethiopia \\ ${ }^{2}$ North Wollo, Zonenal Health Departments, Ethiopia
}

Email address:

workuawo@yahoo.com (W. Awoke),jem.muali@gmail.com (J. Muhammed), abejegedefaw@yahoo.com (G. Abeje)

To cite this article:

Worku Awoke, Jemal Muhammed, Gedefaw Abeje. Institutional Delivery Service Utilization in Woldia, Ethiopia, Science Journal of Public Health. Vol. 1, No. 1, 2013, pp. 18-23. doi: 10.11648/j.sjph.20130101.13

\begin{abstract}
Introduction: Institutional delivery service utilization is essential to improve maternal and child health. However, little is known about institutional delivery service utilization in Woldia, Ethiopia. This study was aimed at assessing institutional delivery service utilization. Methods: Community based cross sectional study was done on 478. Census was carried out in two urban and one rural kebeles (the smallest administrative unit) to identify mothers who gave birth in the past five year. Mothers were selected by systematic random sampling technique. Quantitative data and qualitative data were collected by means of a pretested questionnaire and in-depth interview guide, respectively. Bivariate and multiple logistic regression analysis were done by using SPSS version 20; P-values $\leq 0.05$ were considered as statically significant. Results: The study showed that $48.3 \%$ of institutional delivery service utilization. Mothers' residences, educational status, being informed about place of delivery at their last pregnancy were main factors associated to institutional delivery service utilization. Conclusions: Institutional delivery service in Woldia was low. It is recommended that motivating mothers through formal education and awareness creation about the benefit of institutional delivery service utilization and the risk of home delivery should be given due attention by the local health workers.
\end{abstract}

Keywords: Institutional Delivery Service; Woldia; Ethiopia

\section{Introduction}

Globally, 287000 mothers die from complications of pregnancy and childbirth. Sub-Saharan Africa and Southern Asia accounted for $85 \%$ of the global burden of maternal deaths [1]. The rate of maternal mortality in Ethiopia is one of the highest in the world and estimated 673 maternal deaths per 100,000 live births in 2012 [2]. Most maternal deaths are avoidable, as the health-care solutions to prevent or manage complications are well known. It is particularly important that all births are attended by skilled health professionals, as timely management and treatment can make the difference between life and death [3].

Recent efforts to reduce maternal mortality in developing countries have focused primarily on training and deploying skilled birth attendants and upgrading emergency obstetric care facilities [4]. However, the proportion of birth attended by skilled health workers varies across the regions. Nearly all births in developed countries, $61.9 \%$ in less developed countries, $35.3 \%$ in the least developed countries and $33.7 \%$ births in eastern Africa were attended by skilled health personnel [5].

In Ethiopia institutional delivery service utilization at na- tional level was very low, only $10 \%$ of births were assisted by skilled health care provider. Moreover, sixty one percent of mothers stated that health facility delivery was not necessary and thirty percent stated that it was not customary to deliver at health facility [2]. Previous local studies done in Ethiopia showed that there were variation in skilled birth attendance, $12.3 \%$ in Munisa district [6], 4.3\% of rural mothers and 40\% urban mother in Arsi [7], 12.0\% (20.8\% in urban and $6.8 \%$ in rural mother) in Metekel [8], 13.5\% in North Gondar [9], 12.1\% in Sekela district [10].

Previous studies also showed that institutional delivery service utilization were associated with educational status of mothers and their husbands, income level, preferences of the attentions of their relatives, trust on traditional birth attendants, absence of health problems during pregnancy, antenatal visits during pregnancy, short duration of labor, mothers' place of residence, age, perceived distance to the nearest health facility and transportation costs $[6,7,8,9,10]$. However, little is known about institutional delivery service utilization in Woldia, Ethiopia. This study was aimed at assessing institutional delivery service utilization and associated factors among mothers who gave birth in the past five year prior to this survey. 


\section{Methods}

\subsection{Study Area and Period}

Community based cross sectional study was done by using quantitative and qualitative method of data collection from February to March 2012 in Woldia, Ethiopia. Woldia is located at a distance of $520 \mathrm{~km}$ from Addis Ababa in North Ethiopia which is administratively structured by four rural and three urban kebeles (lowest administrative level) and had a total a total population of 46,139, of whom 23,000 are men and 23,139 women [11].There is one hospital, two health centers and four health posts.

\subsection{Sample Size Determination and Sampling Method}

Sample size was determined using single population proportion formula. The following assumptions were used, the proportion of institutional delivery in Amhara Region according to previously study was $10.2 \%$ [2], $95 \%$ confidence level, $4 \%$ margin of error, multiplying by two for designing effect and additional 10\% allowance for none response rate, making the calculated sample size to be 478 mothers.

Participants for quantitative data were selected by using the following steps. First, two urban and one rural kebeles (the smallest administrative unit) were selected by using simple random sampling technique. Census was carried out in the selected Kebeles to identify mothers who gave birth in the past five year prior to the survey. Then, proportional distribution of the sample size for each selected kebeles was done. Finally, mothers were selected by systematic random sampling method. For qualitative data, key informants were selected conveniently from district health office, district hospital and health centers.

\subsection{Data Collection and Management}

A structured questionnaire was originally prepared in English according to the objective of the study and translated into the local language (Amharic). Sections that showed any discrepancies were revised. Ten females, who completed secondary school education, were recruited for data collection. Data collectors and supervisors were trained mainly on the objective of the study, how to conduct face to face interview and how to keep confidentiality of the information. Then data were collected by using a face-to-face interview after pre-testing in a similar setting of the study area. The data collection processes were supervised daily in the field by health professionals. The collected data were coded and entered in to a statistical package of SPSS version 20. Bivariate and multiple logistic regression analysis were done; P-values $\leq 0.05$ were considered as statically significant. The qualitative data were collected by using in-depth interviews with key informants. After gathering the qualitative data, it was organized manually and thematic analysis was done.

\subsection{Ethical Considerations}

Ethical clearance was obtained from ethical review committee of Bahir Dar University. Letter of permission was obtained from Woldia administration. During data collection, the purposes of the study were clearly explained and informed oral consent was obtained from the selected mothers. Participants were assured of confidentiality of the information and told that they had the right to withdraw from the study at any time during interviewing.

\section{Results}

\subsection{Socio-Demographic Characteristics of Respondents}

From the total of 471 mothers interviewed, 181(38.4\%) and $290(61.6 \%)$ of respondents were rural and urban residents respectively. Twelve (2.5\%), 78(16.6\%), 140(29.7\%), $241(51.1 \%)$ of the respondents were in the age group of 15-19, 20-24, 25-29, and above 30 years, respectively. Majority of mothers, $434(92.1 \%)$ were belong to the Amhara ethnic group. Concerning educational status, 224(47.6\%) of mothers cannot read and write, $47(10 \%)$ can read and write, $105(22.3 \%)$ attended primary education and $95(20.2 \%)$ of the respondents completed secondary education and above. As to occupational status, 334(70.9\%) were house wives, 63 (13.4\%) were farmers, and 74(15.7\%) had other occupations. Regarding marital status, 37(7.9\%) were single, 397(84.3\%) married, 17(3.6\%) divorced, 7(1.5\%) separated, and $13(2.8 \%)$ were widowed (Table 1$)$.

Table 1. Socio-demographic characteristics of the respondents, March 2012. $(N=471)$

\begin{tabular}{|c|c|c|}
\hline Variables & Frequency & Percent \\
\hline \multicolumn{3}{|l|}{ Residence } \\
\hline Rural & 181 & 38.4 \\
\hline Urban & 290 & 61.6 \\
\hline \multicolumn{3}{|l|}{ Age (years) } \\
\hline $15-19$ & 12 & 2.5 \\
\hline $20-24$ & 78 & 16.6 \\
\hline $25-29$ & 140 & 29.7 \\
\hline$\geq 30$ & 241 & 51.2 \\
\hline \multicolumn{3}{|l|}{ Religion } \\
\hline Muslim & 155 & 32.9 \\
\hline Orthodox & 300 & 63.7 \\
\hline Protestant & 16 & 3.4 \\
\hline \multicolumn{3}{|l|}{ Ethnicity } \\
\hline Amhara & 434 & 92.1 \\
\hline Tigrie & 37 & 7.9 \\
\hline \multicolumn{3}{|l|}{ Educational status } \\
\hline Cannot read and write & 224 & 47.5 \\
\hline Can read and write & 47 & 10 \\
\hline Primary & 105 & 22.3 \\
\hline Secondary and above & 95 & 20.2 \\
\hline \multicolumn{3}{|l|}{ Occupational status } \\
\hline House wife & 334 & 70.9 \\
\hline Framer & 63 & 13.4 \\
\hline Others & 74 & 15.7 \\
\hline \multicolumn{3}{|l|}{ Marital status } \\
\hline Single & 37 & 7.9 \\
\hline Married & 397 & 84.3 \\
\hline Divorced & 17 & 3.6 \\
\hline Separated & 7 & 1.5 \\
\hline Windowed & 13 & 2.8 \\
\hline
\end{tabular}




\subsection{Obstetric Characteristics of Respondents}

Two hundred forty eight 248 (52.7\%) of the respondents were 15-19 years at their first pregnancy, 164 (34.8\%) 20-24 and $50(10.6 \%)$ of the respondents were 25-29 years old. Regarding age of mother during the last birth, $50(10.6 \%)$ were 15-19 years, 125 (26.5\%) were 20-24 years, $113(24 \%)$ mothers were $25-29$ years, and $183(38.9 \%)$ mothers were older than 30 years. Majority 419 (89\%) of the respondents had a history of antenatal care services. Three hundred forty three $(72.8 \%)$ of respondents reported that they obtained information about complication during delivery and 364 (77.3\%) of the respondents reported that they were informed about the place of delivery during their antenatal visits (Table 2).

Table 2. Obstetric characteristics of the respondents, March 2012.

\begin{tabular}{|c|c|c|}
\hline Variables & Frequency & Percent \\
\hline \multicolumn{3}{|c|}{ Age at first pregnancy } \\
\hline $15-19$ & 248 & 52.7 \\
\hline $20-24$ & 164 & 34.8 \\
\hline $25-29$ & 50 & 10.6 \\
\hline$\geq 30$ & 9 & 1.9 \\
\hline \multicolumn{3}{|c|}{ Age at last pregnancy } \\
\hline $15-19$ & 50 & 10.6 \\
\hline $20-24$ & 125 & 26.5 \\
\hline $25-29$ & 113 & 24 \\
\hline$\geq 30$ & 183 & 38.9 \\
\hline \multicolumn{3}{|l|}{ Gravidity } \\
\hline 1 & 125 & 26.5 \\
\hline $2-4$ & 305 & 64.8 \\
\hline$\geq 5$ & 41 & 8.7 \\
\hline \multicolumn{3}{|l|}{ Parity } \\
\hline 1 & 126 & 26.8 \\
\hline $2-4$ & 319 & 67.7 \\
\hline$\geq 5$ & 26 & 5.5 \\
\hline \multicolumn{3}{|l|}{ ANC visit } \\
\hline Yes & 419 & 89 \\
\hline No & 52 & 11 \\
\hline \multicolumn{3}{|c|}{ Frequency of ANC visits } \\
\hline $1-3$ & 181 & 43.2 \\
\hline 4 & 238 & 56.8 \\
\hline \multicolumn{3}{|c|}{ Informed about delivery complications } \\
\hline Yes & 343 & 81.9 \\
\hline No & 73 & 17.4 \\
\hline I don't remember & 3 & 0.7 \\
\hline \multicolumn{3}{|c|}{ Informed about place of delivery } \\
\hline Yes & 364 & 86.9 \\
\hline No & 55 & 13.1 \\
\hline
\end{tabular}

\subsection{Institutional Delivery Service Utilization}

Majority, 470 (99.8\%) of the respondents reported that the availability of health facility in the nearby to their home. However, 132 (28\%) of respondents did not know whether that health facility provide delivery service or not. Among 471 respondents, $227(48.3 \%)$ gave their last birth in health institutions, $243(51.7 \%)$ gave birth at home. Out of the total home deliveries, $22.5 \%, 10.4 \%$ and $7.4 \%$, of the deliveries were assisted by family members and relatives, untrained traditional birth attendants, trained traditional birth attendants, respectively (Table 3).

Table 3. Institutional delivery service Utilization in Woldia, March 2012March 2012

\begin{tabular}{lll}
\hline Variable & Frequency & Percent \\
& & \\
\hline & & \\
Do you know that the nearby & & \\
Health facility is providing & & \\
delivery service? & 333 & 70.7 \\
Yes & 132 & 28 \\
No & 6 & 1.3 \\
I don't know & & \\
& & \\
& & \\
Place of delivery of the last pregnancy & & \\
Home & 243 & 51.6 \\
Health facility & 227 & 48.2 \\
Others & 1 & 0.2 \\
& & \\
& & \\
Had complication during last & & \\
delivery among home \\
deliveries (N=243)
\end{tabular}

The most common reasons for home delivery were precipitate labor $47.9 \%$, smooth and short labor $42 \%$ and labor at night $14 \%$ (Figure 1). Most mothers who gave birth at home reported that they experienced complications, Retained placenta $(39 \%)$ prolonged labor $(27.3 \%)$, excessive vaginal discharge and loss of consciousness (Figure 2). 


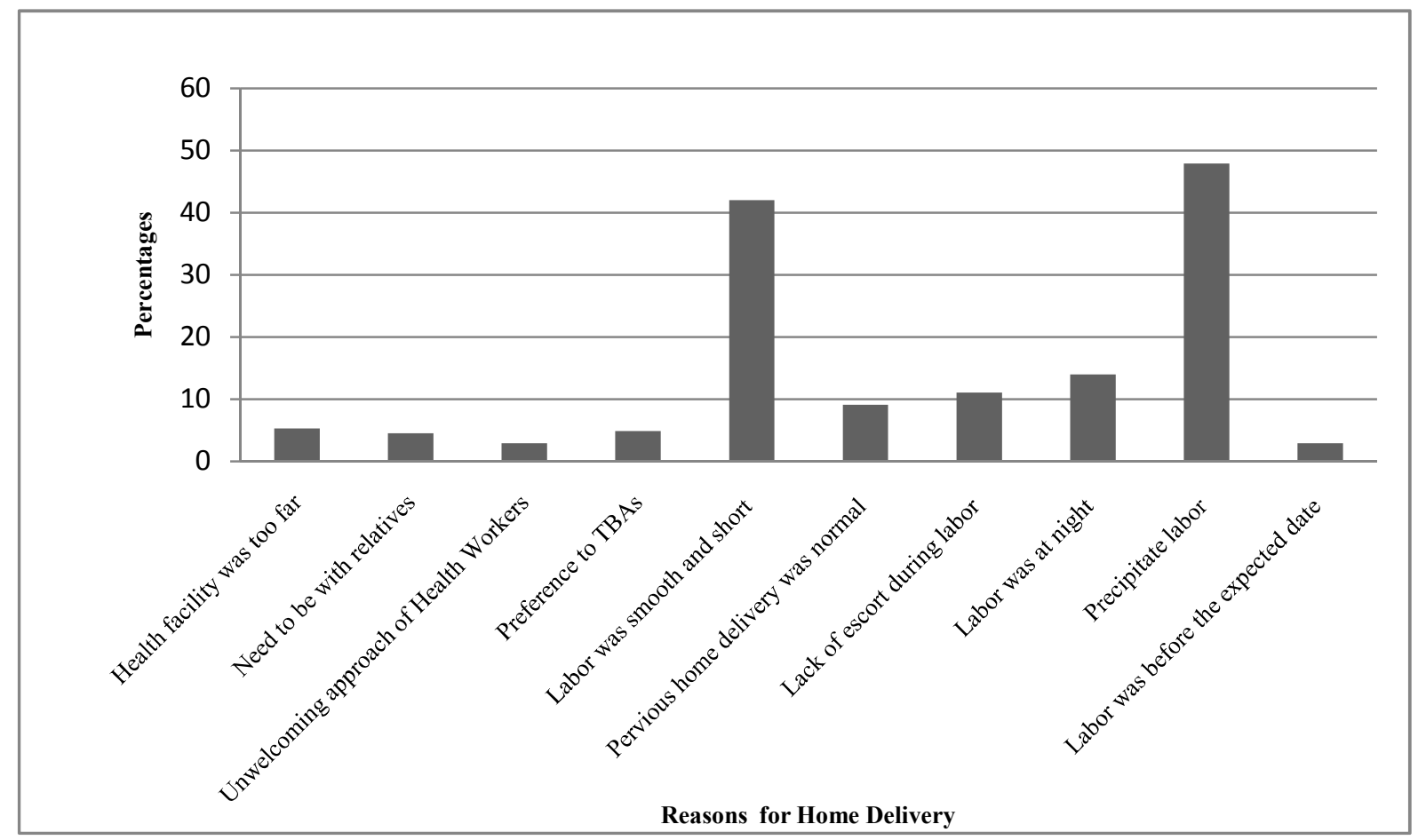

Figure 1. Reasons for home delivery as mentioned mothers in Woldia, March 2012.

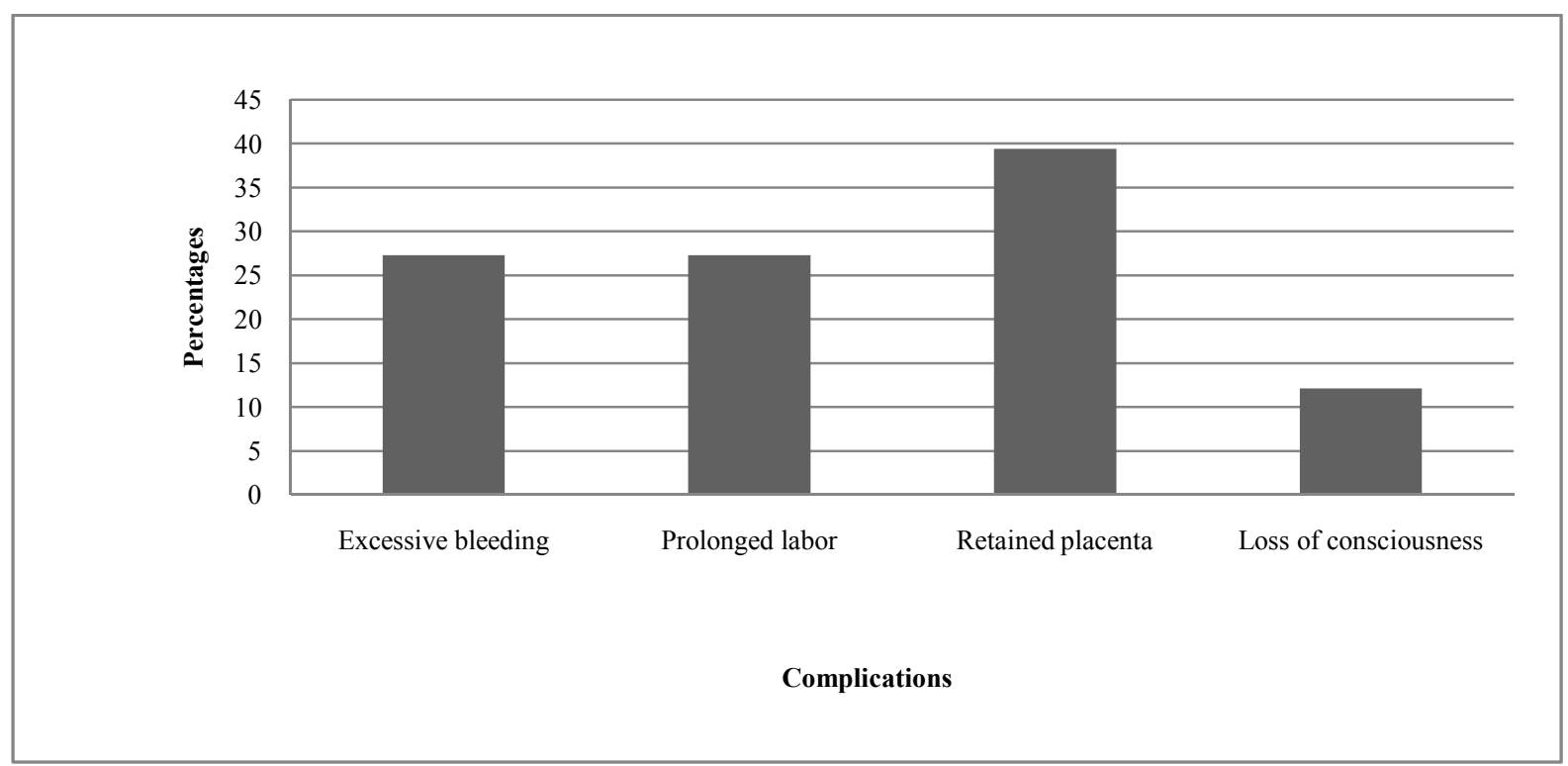

Figure 2. Types of complications faced during home delivery as reported by mothers who gave birth at home in Woldia, March 2012

\subsection{Factors Associated with Institutional Delivery Service Utilization}

Bivariate analysis was carried out to examine factors associated with institutional delivery service. The socio demographic variables which showed statistically significant association were place of residence, mothers' education status and occupation, husband's education and occupation ( $p$-value $\leq 0.05)$. The obstetric variables that showed statistically significant association ( $p$-value $\leq 0.05$ ) were mothers' age at first pregnancy, frequency of antennal care visits and obtaining information on the importance of institutional delivery during their last pregnancy.

Finally, multivariate logistic regression was carried out to predict institutional delivery service utilization with variables that were statistically associated during the bivariate analysis $\mathrm{p}$-value $\leq 0.05$ ). Mothers' residence, educational status, obtaining information on the importance of institutional delivery during their last pregnancy and knowledge of mothers about institutional delivery services were significantly associated with the utilizations of institutional 
delivery service. The result showed that mothers who live in rural areas were less likely to utilize institutional delivery service than mothers from urban areas $(\mathrm{AOR}=0.032$ and $95 \% \mathrm{CI}=0.004-0.25)$. Mothers who had secondary and above educational level were more likely to utilize institutional delivery service than those who cannot read and write (AOR 15 and 95\% $\mathrm{CI}=4.31-54.8$ ). Mothers who were not informed about place of delivery were less likely to utilize institutional delivery service than informed ones ( $\mathrm{AOR}=0.081$ and $95 \% \mathrm{CI}=0.007-0.99$ ).

Mothers who were less knowledgeable less knowledgeable about institutional delivery service utilization and delivery complications were less likely to utilize delivery service than mothers who had adequate knowledge $(\mathrm{AOR}=0.20$ $95 \% \mathrm{CI}=(0.046-0.89)$ (Table 4).

Table 4. Associations of socio- demographic and obstetric characteristics of respondents with institutional delivery service utilization in Woldia, March 2012 March 2012.

\begin{tabular}{|c|c|c|c|c|}
\hline Variables & \multicolumn{2}{|c|}{ Institutional delivery } & Crud OR 95\% CI & $\begin{array}{l}\text { Adjusted OR } \\
\text { 95\% CI } \\
\end{array}$ \\
\hline \multicolumn{5}{|l|}{ Residence } \\
\hline Rural & 15 & 166 & $0.034(0.018-0.060)$ & $0.032(0.004-0.25)$ \\
\hline Urban & 210 & 78 & 1.00 & 1.00 \\
\hline \multicolumn{5}{|l|}{ Educational status } \\
\hline Cannot read and write & 34 & 190 & 1.00 & 1.00 \\
\hline Can read and write & 31 & 16 & $10.82(5.34-21.91)$ & $6.86(2.19-21.47)$ \\
\hline Primary education & 74 & 31 & $13.34(7.65-23.25)$ & $9.76(4.03-23.64)$ \\
\hline Secondary and above & 86 & 9 & $53.39(24.53-116)$ & $15(4.31-54.8)$ \\
\hline \multicolumn{5}{|l|}{$\begin{array}{l}\text { Informed } \\
\text { about place of } \\
\text { delivery during }\end{array}$} \\
\hline last pregnancy & 218 & 146 & 1.00 & 1.00 \\
\hline Yes & 2 & 53 & $0.025(0.006-0.10)$ & $0.081(0.0007-0.991)$ \\
\hline \multicolumn{5}{|l|}{ No } \\
\hline \multicolumn{5}{|l|}{$\begin{array}{l}\text { Knowledge of } \\
\text { mothers about } \\
\text { institutional }\end{array}$} \\
\hline delivery services & 220 & 175 & 1.00 & 1.00 \\
\hline Knowledgeable & 5 & 71 & $0.056(0.022-0.14)$ & $0.20(0.046-0.89)$ \\
\hline Not knowledge & & & & \\
\hline
\end{tabular}

\section{Discussion}

This study showed that institutional delivery service utilization in the study area was low [48.3\%]. This finding is consistent with other findings in Ethiopia; EDHS 2011[2], in the urban Arsi Zone [7] and in Metekel [8]. This finding was also supported with the qualitative data findings. The key-informant said that; "Like any Ethiopian region, proportion of mothers who use institutional delivery service in Woldia town is low. Most mothers in our town (Woldia) gave birth at home without the help of skilled birth attendants."

The result of the present study depict that place of residence had significant association with institutional delivery service utilization. The rural mothers were less likely to give birth at health facility compared to rural mothers. This finding was consistent with other research findings reported in Ethiopia, find dings of Ethiopia demographic and health surveys of year 2011 [2], Metekel [8] and Gondar [9]. The reason might be that urban mothers tend to have better access to health facilities and other promotional services than rural mothers. On the other hand, rural mothers might be tied with strong cultural and traditional belief and lack awareness about the benefit of institutional delivery servic- es.

Mothers who had educational level of secondary and above were fifteen times more likely to utilize institutional delivery service than mothers who cannot read and write. This finding is consistent with that of some previous studies which showed that educational status of mothers to be the most significant associated factors for utilization of institutional delivery service [2, 7-9]. There are a number of reasons why educational status of mothers has significant positive relationship with utilization of institutional delivery service. Education may enhance female autonomy; thus increasing mothers' ability to make decisions regarding their utilization of institutional delivery service. Education also increases the knowledge of delivery care, thus increasing the demand for utilization of institutional delivery service.

Study conducted in Ethiopia revealed that antenatal care visit could increase utilization of institutional delivery service $[8,9]$. In this study $89 \%$ of mothers received antenatal care services for the last pregnancy. However, antenatal care visit did not show statistically significant association with institutional delivery service utilization. This finding was also supported by findings of the qualitative data. One key informant said that; "All mothers who had antenatal care visit did not give birth at health facility. This is due to the 
fact that, during antenatal care, mothers did not get enough information about the risk of home delivery. On other hand, mothers can come for antenatal care by themselves, but they could not come when they are in labor because they need the help of others. Therefore, the coverage of institutional delivery is lower than other maternal health care services."

Majority of mothers preferred home delivery. The most common reasons for home delivery were precipitate labor, smooth and short labor, labor was at night, normal home delivery in previous time, preference to traditional birth attendants and relatives. Similar study from Arisi Zone revealed that the main reasons for mothers to prefer home delivery was short labor 54.8\% [7]. Another study in Gondar identified that the main reasons for home delivery were smooth and short labor (42\%), need to be attended with relatives during labor $(44.7 \%)$, trusting traditional birth attendants and cultural belief (55.3\%) [9].

This study showed that mothers who delivered at home had faced birth complications. Mothers who delivered at home reported complications such as retained placenta (39.4\%), prolonged labor $(27.3 \%)$, excessive vaginal bleeding $(27.3 \%)$ and loss of consciousness (12\%).This finding is similar with the result reported from North Gondar, which revealed that $41.1 \%, 24.2 \%$ and $18.6 \%$ of mothers who gave birth at home had excessive vaginal bleeding, prolonged labor and retained placenta respectively [9].

Key informant said that; "Mothers experienced complications when they delivered at home, such as retained placenta, prolonged labor, excessive vaginal bleeding and fetal distress. Mothers come to health institutions after they have developed complications."

\section{Conclusion}

This study showed that there was low institutional delivery service utilization in Woldia. The socio-demographic characteristics of mothers, residence, educational status, being informed about place of delivery and knowledge of mothers about delivery service utilization were the common associated factors on the utilization of institutional delivery service in Woldia. Even though $89 \%$ of mothers received antenatal care, only $48.3 \%$ of those were delivered at health facility depicting that there was a gap between antenatal care service and delivery service utilization. Based on our findings, it is important to increase girls education to improve institutional delivery service utilization. Health education for mothers about benefits of institutional delivery service utilization and risks of home delivery should be also given due attention by the local health workers to increase institutional delivery service utilization.

\section{Limitation of the study}

The limitations of this study may include the following. This cross sectional study by its very nature cannot establish cause and effect relationship. Some of the data like antenatal visit, being informed about place of delivery and types of complication they faced during home delivery were based on mothers response.

\section{Acknowledgements}

We would like to thank Bahir Dar University and GAMBY College of Medical Sciences. We are also grateful for the cooperation of data collectors and the study participants.

\section{References}

[1] World Health Organization: Trends in Maternal Mortality: 1990 to 2010 Estimates developed by WHO, UNICEF, UNFPA and The World Bank. Geneva; 2012.

[2] Central Statistical Agency [Ethiopia] and ICF International: Ethiopia Demographic and Health Survey 2011: Addis Ababa. Ethiopia and Calverton, Maryland, USA: Central Statistical Agency and ICF International; 2012.

[3] World health organization: Maternal mortality (2012) url:http://www.who.int/mediacentre/factsheets/fs348/en/inde x.html

[4] Prata N, Passano P, Rowen T, Bell S, Walsh J and Potts M: Where There Are (Few) Skilled Birth Attendants. Journal of health nutrition, population and Nutrition. 2011 April; 29(2): 81-91.

[5] Department of Reproductive Health and Research: Proportion of births attended by a skilled health worker By World Health organization, Geneva; 2008 updates. Url: http://www.who.int/reproductivehealth/publications/materna 1_perinatal_health/2008_skilled_attendant.pdfl:

[6] Amano A, Gebeyehu A and Birhanu Z: Institutional delivery service utilization in Munisa Woreda, South East Ethiopia: a community based cross-sectional study. BMC Pregnancy and Childbirth 2012, 12:105.

[7] Abera M, G/mariam A, and Belachew T: predictors of safe delivery service utilization in Arsi zone, south-east Ethiopia. Ethiop journal of Health Sciences. 2011; Vol. 21, Special Issue: 101-113.

[8] Tura G, G/Mariam : Safe delivery service utilization in Metekel Zone , North West Ethiopia. Ethiop journal of Health Sciences. 2011; 17(4): 231-222

[9] Nigussie M, Haile Mariam D, Mitike G: Assessment of safe delivery service utilization among Mothers of childbearing age in north Gondar Zone, North West Ethiopia. Ethiopian journal of Health Development .2004;18(3):145-152

[10] Teferra AS, Alemu FM, Woldeyohannes SM: Institutional delivery service utilization and associated factors among mothers who gave birth in the last 12 months in Sekela District, North West of Ethiopia: A community - based cross sectional study. BMC Pregnancy and Childbirth 2012, 12(74):1-11.

[11] Central Statistical Agency [Ethiopia]. 2007 Population and housing census results of the Amhara Region, Addis Ababa. 